\title{
Severe Pulmonary Hypertension in Acute \\ Pulmonary Thromboembolism: More Common than Expected?
}

\author{
Mahsa Behnemoon \\ Urmia University of Medical Sciences \\ Elham Laleh \\ Urmia University of Medical Sciences \\ Amin Sedokani ( $\square$ a.sedokani@gmail.com ) \\ Urmia University of Medical Sciences \\ Ayatay Bahrami \\ Urmia University of Medical Sciences
}

\section{Research Article}

Keywords: Pulmonary embolism, systolic pulmonary artery pressure, right ventricular dysfunction, pulmonary artery hypertension, tricuspid regurgitation severity

Posted Date: December 7th, 2020

DOI: https://doi.org/10.21203/rs.3.rs-112724/v1

License: (c) (i) This work is licensed under a Creative Commons Attribution 4.0 International License. Read Full License 


\section{Abstract}

Background: Pulmonary embolism (PE) is a relatively common health problem and the third most common cause of cardiovascular death with a 15-20 percent mortality rate. Severe pulmonary arterial hypertension is not common in acute forms of the disease, and we usually expect only mild to moderate degrees of PAH in these patients. However, we have encountered numerous cases of severe pulmonary hypertension after acute PE in our practice. In this study, we aimed to evaluate the echocardiographic findings of patients admitted with documented PE in a 5 years study of two heart centers of Urmia.

Methods: In this retrospective study, the data of 183 patients with a definitive diagnosis of acute pulmonary embolism based on pulmonary CT angiography have entered the study.

Results: Of the 183 cases diagnosed with pulmonary embolism with an average age of 61.15 years, $45.4 \%$ were male and $54.6 \%$ were female. Shortness of breath, chest pain, and hemoptysis were seen in $88 \%, 49.2 \%$, and $13.1 \%$ of patients, respectively. Tachypnea and tachycardia were the most common clinical findings with a frequency of $54.1 \%$. Echocardiographic findings of right ventricular enlargement and right ventricular dysfunction were observed in $66.7 \%$ and $67.8 \%$ of patients, respectively and $42.1 \%$ of patients had severe pulmonary hypertension. We found a significant relationship between pulmonary artery pressure severity and shock state as well as in-hospital mortality. While only 3 patients out of 64 cases $(4 \%)$ with normal or mildly elevated pulmonary artery pressure died in their hospital stay period, the mortality rate was $28.5 \%$ in patients with moderate or severe pulmonary artery hypertension ( $p$ value $=0.002$ ).

Conclusion: we found a relatively high frequency of severe pulmonary artery hypertension in patients admitted with the definite diagnosis of acute pulmonary embolism and there was a significant correlation between pulmonary artery pressure severity and shock state, as well as in-hospital mortality. So, echocardiographic findings including right ventricular systolic pressure and TR velocity may have additional prognostic value in the decision making of acute PE patients and could be helpful in reducing in-hospital mortality of this complex illness provided being included in prognostic models of acute PE, based on future studies.

\section{Introduction}

Pulmonary embolism (PE) is the third most common cause of cardiovascular death (after myocardial infarction and stroke) that mostly originates from deep vein thrombosis of the lower limbs. ${ }^{1-3}$ Genetic and acquired factors are involved in the development of venous thromboembolism. Obesity, smoking, oral contraceptives, postmenopausal hormone replacement therapy, surgery, cancer, systemic arterial hypertension, and chronic obstructive pulmonary disease are among the most common acquired underlying factors. ${ }^{3,4}$ The incidence of pulmonary embolism in the United States is estimated at 1 in 1,000 people, although actual rate appears to be higher as many patients remain undiagnosed in some 
conditions. The mortality rate from pulmonary embolism in the first 3 months after diagnosis reaches more than $15 \%$ and is usually resulting from right ventricular failure. ${ }^{5-7}$

Patients with pulmonary embolism present with various symptoms. According to some studies, $73 \%$ of these patients suffer from dyspnea, whereas chest pain, cough, and hemoptysis are present in 66, 37 and 13 percent, respectively. ${ }^{8}$ In physical examination, tachypnea is reported to be the most clinical finding with the prevalence of $70 \%$, followed by tachycardia which is seen in $30 \%$ of patients. In severe cases of pulmonary embolism, patients may present with shock state or hemodynamic instability. ${ }^{9}$ Because of these nonspecific clinical manifestations, diagnostic tests play an essential role in clinical decision makings. Helpful initial work up besides history and physical examination, includes chest X ray, electrocardiography, echocardiography, plasma D-dimer level (high sensitivity but low specificity) evaluation, lung computed tomography angiography scan (sensitivity $53 \%-100 \%$, specificity $83 \%-100 \%$ ) and perfusion ventilation lung scintigraphy. However, pulmonary angiography remains the gold standard tool of the diagnosis of $\mathrm{PE}$ in the ambiguous cases. ${ }^{8-11} \mathrm{It}$ is believed that patients with systolic blood pressure less than $90 \mathrm{~mm} \mathrm{Hg}$, blood pressure drop more than or equal to $40 \mathrm{~mm} \mathrm{Hg}$ for more than 15 minutes, or shock state resulting from overt right ventricular failure are at the highest risk of early mortality. ${ }^{12}$ Besides, Right ventricular function is an important prognostic factor for pulmonary embolism. $3,12,13$ In patients with stable hemodynamics and normal blood pressure, presence of moderate to severe right ventricular dysfunction in bedside echocardiography is a potential sign of increased short-term and long-term mortality. ${ }^{14,15}$ However the appropriate strategy for the management of these patients is still controversial. ${ }^{16,17}$ Echocardiography is a useful tool in the assessment of right ventricular pressure and function with the sensitivity of $90 \%$ in detecting massive PEs with elevated pulmonary artery pressure (PAP). In these patients, right ventricular dilatation and McConnell's sign (midfree wall akinesis but normal apical motion) as well as D-shaped left ventricle due to the displacement and flattening of inter-ventricular septum, are among the most common diagnostic echocardiographic clues for significant PE. ${ }^{18,19}$ Although at least 4 percent of cases presented by acute episode of PE have been reported to show symptomatic chronic pulmonary hypertension at 2 years follow up, ${ }^{20}$ but the prevalence of acute severe pulmonary hypertension following acute PE is assumed to be low because of the right ventricle geometry, which cannot tolerate acute severe pressure rise and becomes dilated and dysfunctional before additional rise of the pulmonary artery pressure (PAP) beyond moderate degrees. While chronic pulmonary hypertension stimulates adaptive changes in the RV myocardium that help maintain RV stroke volume, this does not occur in the acute setting and in normal individuals, the RV is unable to acutely generate a mean pressure $>40 \mathrm{mmHg}$ (which is equal to systolic PAP of $60^{\mathrm{mmhg}}$ ), and stroke volume decreases linearly as RV afterload increases. ${ }^{21}$ Despite these facts, we have encountered numerous cases of severe pulmonary hypertension after acute PE with or without hemodynamic instability in our practice. Besides, systolic pulmonary artery pressure (sPAP) measured in transthoracic echocardiography is not a well established marker of risk stratification in acute PE in multiple prognostic models. Hence we decided to design this study with the aim of evaluating the clinical features and echocardiographic findings (with more focus on the parameter of sPAP for prediction of mortality) of our 
patient population who were admitted to our general educational hospitals with the definite diagnosis of PE during 2014-2019.

\section{Methods}

In this descriptive-analytical cross-sectional study, 321 patients who were admitted to Urmia educational hospitals between 2014 and 2019 with the final diagnosis of PE were collected, of which 113 were excluded due to the absence of recent pulmonary CT angiographic data, 7 due to the diagnosis of chronic thromboembolic pulmonary hypertension (CTEPH) according to the patients previous admission information, and 18 due to secondary diagnosis of PAH (8 with decompensated heart failure, 6 with severe left heart valvulopathy and 4 with underling collagen vascular disease). Finally, 183 patients with a definitive diagnosis of pulmonary embolism based on pulmonary CT angiography were eligible for our study. Clinical history and initial vital signs, echocardiographic findings regarding right ventricular size and function, tricuspid regurgitation (TR) severity and pulmonary artery systolic pressure, as well as the final status of the patients (discharge or death) were extracted from each patient's hospital record. Systolic PAP below $30^{\mathrm{mmhg}}$ was considered normal, while severe PAH was defined as SPAP above $60^{\mathrm{mmh}}$. Finally, gathered data were analyzed to verify any correlation between various echocardiographic findings and in-hospital mortality.

\section{Data analysis}

Descriptive statistics for the relevant baseline characterization of PE patients were provided with mean and standard deviation or corresponding frequency. Continuous variables, which did not follow a normal Gaussian's distribution, were compared with the help of Wilcoxon-Whitney-U-test. Normal distributed continuous variables were compared using Stu-dents' T-test, as appropriate. Data analysis was performed using SPSS22 software and $P$ values less than 0.05 were considered significant.

\section{Results}

\section{Clinical features}

In this study $45.4 \%$ (83) of patients were male and $54.6 \%$ (100) were female. The mean age \pm SD was $61.15 \pm 18.98$ years. Dyspnea, chest pain and hemoptysis were present in $88 \%, 49.2 \%$ and $13.1 \%$ of patients, respectively. Syncope was the first presentation symptom in $13(7.1 \%)$ patients, whereas in 68 $(37.2 \%)$ cases cardiogenic shock (SBP[1] $<90^{\mathrm{mmHg}}$ or MAP[2] $<60^{\mathrm{mmHg}}$ ) was recorded. Tachycardia and tachypnea were the most clinical findings, both positive in $54.1 \%$ of our patient population (table-1). We found in-hospital mortality rate of $20.2 \%$ (37 patients) in our study, and the remaining $(79.8 \%)$ were discharged from the hospital with significant symptoms improvement.

\section{Echocardiography}


In echocardiographic assessment, our results indicated that the mean left ventricle ejection fraction (LVEF) for patients was $49.21 \pm 9.29 \%$ with the mean PAP of $50.82 \pm 22.88^{\mathrm{mmHg}}\left(12-125^{\mathrm{mmHg}}\right)$. Tricuspid regurgitation (TR) was not observed in $12.6 \%$ of patients; however, $35.5 \%$ had mild, $28.4 \%$ had moderate and $23.5 \%$ had severe TR in transthoracic echocardiography examination. Of 37 patients $(20.2 \%)$ who died during their hospital stay period, 2 had normal tricuspid valve, 5 had mild regurgitation, 13 had moderate and 17 had severe TR. Out of 146 discharged patients, the mentioned parameters were 21, 60, 42 and 23 respectively. There was a significant relationship between TR severity and hospital outcomes ( $p$-value $=0.007)$. (table-2)

Right ventricular (RV) enlargement was observed in total $122(66.7 \%)$ patients, and $81.1 \%$ of deceased cases had some degrees of right ventricular dysfunction; whereas only 7 patients without RV dysfunction died during their admission period. Among discharged cases, $64.4 \%$ reported to have at least moderate right ventricular dysfunction which indicated a significant correlation between in hospital outcomes and right ventricular dysfunction in patients with acute pulmonary embolism ( $p$-value=0.03). (table-2)

The mean SPAP was $48.20 \pm 22.85^{\mathrm{mmHg}}$ in patients discharged from the hospital and $61.16 \pm 20.10^{\mathrm{mmHg}}$ in patients who died in hospital, which showed a significant relationship between sPAP and hospital outcomes in patients with acute PE ( $p$-value=0.03).

Also we found a significant difference between the percentage of LVEF and in hospital outcomes, where the mean LVEF in patients discharged from the hospital was $49.90 \pm 9.55 \%$ versus $46.49 \pm 7.71 \%$ in patients who died ( $p$-value $=0.04$ ).

There was a significant relationship between pulmonary artery pressure severity and shock state, as well as in hospital mortality. In 77 patients (42.1\%), severe pulmonary hypertension was seen, of which 37 cases had initial manifestation of cardiogenic shock and 23 patients died; While only 3 patients out of 47 cases with normal pulmonary pressure and 11 patients out of 42 cases with moderate PAP died in hospital ( $p$-value $=0.002$ ), and the frequency of shock state in the patients without severe PAH was $29.2 \%$ comparing to $48 \%$ in those with severe PAH ( $p$-value $=0.006$ ) (table-3).

\section{Footnote:}

[1] Systolic Blood Pressure

[2] Mean Arterial Pressure

\section{Discussion}

We found relatively high frequency of severe pulmonary hypertension in patients admitted with the definite diagnosis of acute pulmonary embolism, and there was a significant correlation between pulmonary artery pressure severity and shock state, as well as in-hospital mortality. In this study, 183 patients with pulmonary embolism with a mean age of $61.15 \pm 18.98$ years were enrolled, of which $54.6 \%$ 
were female. Multiple previous studies were consistent with ours regarding the highest percentage of patients being female and in their middle-age. ${ }^{22-24}$ We found the mortality rate of $20.2 \%$ in our pulmonary embolism patients during hospitalization which was relatively the same as other large studies to date. ${ }^{5-7,22}$

The most common clinical symptoms and signs of our patients were dyspnea (88\%), tachycardia (54.1\%), tachypnea (54.1\%), chest pain (49.2\%), and shock state (37.2\%). The initial presentation of syncope and hemoptysis were less common ( $7.1 \%$ and $13.1 \%$, respectively). Almost in line with our study, Bajaj et al reported the most common clinical symptoms of their patients as dyspnea (72\%), tachypnea (39\%), chest pain (38\%), tachycardia (32.6\%), cough (19\%), syncope (6\%), and hemoptysis $(4 \%) .{ }^{25}$ In the study of Grifoni et $\mathrm{al}^{26}$ patients with late hemodynamic dysfunction following PE had $10 \%$ shock and $5 \%$ in hospital mortality, which was lower than our statistics possibly due to the relatively older age and smaller sample size of our study.

In our echocardiographic findings right ventricular enlargement and right ventricular dysfunction were seen in $66.7 \%$ and $67.8 \%$ of patients, respectively. While $75.4 \%$ of our cases (138 patients) had elevated PAP (more than $30 \mathrm{~mm} \mathrm{Hg}$ ) during their acute episode of PE, we found 77 patients (42.1\%) with severe pulmonary hypertension (sPAP above $60^{\mathrm{mmhg}}$ ). In the study of Kurnicka et $\mathrm{al}^{24}$ in Poland in 2016, the echocardiographic results of their PE patients revealed right ventricular enlargement in $27.4 \%$ and SPAP > $30 \mathrm{mmHg}$ in $46.6 \%$ of patients which was less common compared to our study, may be due to the differences in the study sample size or even baseline characteristics of the patients.

Based on Jennifer Mathieu's 2008 study, the normal right ventricle could not produce an average pressure above $40^{\mathrm{mmHg}}$ in acute conditions, and stroke volume decreases linearly as RV afterload increases. ${ }^{21}$ Also, according to the study of Wood KE in 2002, TR velocity greater than 3.7 (equivalent to $55^{\mathrm{mmHg}}$ systolic PAP) in acute PE indicates previous underlying pulmonary disease such as chronic thromboembolism ${ }^{28}$. Based on another meta-analysis, risk of severe PAH and CTEPH following pulmonary embolism was reported to be between $0.5-2.3$ percent. ${ }^{29}$ Nevertheless, in $42.1 \%$ of our patients who presented with acute symptoms of $\mathrm{PE}$, we found systolic pulmonary pressure of $\geq 60 \mathrm{mmHg}$, which is inconsistent with the previously mentioned studies. We thought this discrepancy could be resulted from the occurrence of recurrent sub-acute pulmonary embolism or even presence of the underlying pulmonary disease in some patients, despite reviewing all patients' old documents. Following echocardiography examination of these patients at least three months after effective treatments as well as lung perfusion scans (if pulmonary hypertension persists) should be done in order to detect any underlying CTEPH and confirm these hypothesis in the future studies. Therefore gathering all these data together, we suggest large cohort studies with multi logistic regression models to investigate whether systolic PAP and TR velocity could be important markers of increased mortality in patients suffering from acute pulmonary embolism and be included in risk stratification models of this complex illness.

\section{Conclusion}


We found relatively high frequency of severe pulmonary hypertension in patients admitted with the definite diagnosis of acute pulmonary embolism and there were a significant correlation between pulmonary artery pressure severity and shock state, as well as in-hospital mortality. So, echocardiographic findings including right ventricular systolic pressure and TR velocity may have additional prognostic value in decision making of acute PE patients and could be helpful in reducing inhospital mortality of this complex illness provided being included in prognostic models of acute PE based on future studies.

\section{Declarations}

\section{Ethical considerations}

All information was confidential and was extracted without mentioning identifiable details, and ethical issues regarding the observance of patients 'rights and the confidentiality of patients' information were observed, and patients entered the software based on identification codes. The special approved ethical code for the study from ethics committee of Ministry of Health of Iran is IR.UMSU.REC.1397.055. The investigation has met the principles outlined in the Declaration of Helsinki.

\section{Consent for publication}

Not applicable.

\section{Availability of data and materials}

The datasets used and/or analyzed during the current study are available from the corresponding author on reasonable request.

\section{Competing interests}

All of authors report no kind of conflict of interests in this study.

\section{Funding}

This study was funded by Urmia University of Medical Sciences and there is no other organizational or governmental funding.

\section{Authors' Contributions}


All of the authors in this study have contributed equally in design, performance, data collection, analysis, and writing and review of the manuscript.

\section{Acknowledgements}

We would like to thank the health care personnel of Taleghani hospital, specially the cardiology ward personnel.

\section{References}

1. Kistner R, Ball J, Nordyke R, Freeman G. Incidence of pulmonary embolism in the course of thrombophlebitis of the lower extremities. The American Journal of Surgery. 1972;124(2):169-176.

2. Kearon C, Julian JA, Math M, Newman TE, Ginsberg JS. Noninvasive diagnosis of deep venous thrombosis. Annals of internal medicine. 1998;128(8):663-677.

3. Tapson VF. Acute pulmonary embolism. N Engl J Med. Mar 6 2008;358(10):1037-52. doi:10.1056/NEJMra072753

4. Heit JA, Silverstein MD, Mohr DN, Petterson TM, O'Fallon WM, Melton LJ. Risk factors for deep vein thrombosis and pulmonary embolism: a population-based case-control study. Archives of internal medicine. 2000;160(6):809-815.

5. Alpert JS, Smith R, Carlson J, Ockene IS, Dexter L, Dalen JE. Mortality in patients treated for pulmonary embolism. JAMA. Sep 27 1976;236(13):1477-80.

6. Carson JL, Kelley MA, Duff A, et al. The clinical course of pulmonary embolism. N Engl J Med. May 7 1992;326(19):1240-5. doi:10.1056/NEJM199205073261902

7. Kostadima E, Zakynthinos E. Pulmonary embolism: pathophysiology, diagnosis, treatment. Hellenic J Cardiol. 2007;48(2):94-107.

8. Stein PD, Gottschalk A, Sostman HD, et al. Methods of prospective investigation of pulmonary embolism diagnosis III (PIOPED III). Elsevier; 2008:462-470.

9. Meyer G. Effective diagnosis and treatment of pulmonary embolism: Improving patient outcomes. Archives of cardiovascular diseases. 2014;107(6-7):406-414.

10. Stein PD, Terrin ML, Hales CA, et al. Clinical, laboratory, roentgenographic, and electrocardiographic findings in patients with acute pulmonary embolism and no pre-existing cardiac or pulmonary disease. Chest. 1991;100(3):598-603.

11. Kelley MA, Carson JL, Palevsky HI, Schwartz JS. Diagnosing pulmonary embolism: new facts and strategies. Annals of internal medicine. 1991;114(4):300-306.

12. Pruszczyk P, Bochowicz A, Torbicki A, et al. Cardiac troponin T monitoring identifies high-risk group of normotensive patients with acute pulmonary embolism. Chest. 2003;123(6):1947-1952.

13. Meyer T, Binder L, Hruska N, Luthe H, Buchwald AB. Cardiac troponin I elevation in acute pulmonary embolism is associated with right ventricular dysfunction. Journal of the American College of 
Cardiology. 2000;36(5):1632-1636.

14. Sanchez O, Trinquart L, Colombet I, et al. Prognostic value of right ventricular dysfunction in patients with haemodynamically stable pulmonary embolism: a systematic review. European heart journal. 2008;29(12):1569-1577.

15. Horlander KT, Leeper KV. Troponin levels as a guide to treatment of pulmonary embolism. Current opinion in pulmonary medicine. 2003;9(5):374-377.

16. Lankeit M, Friesen $D$, Aschoff $\mathrm{J}$, et al. Highly sensitive troponin T assay in normotensive patients with acute pulmonary embolism. European heart journal. 2010;31(15):1836-1844.

17. Giannitsis E, Müller-Bardorff M, Kurowski V, et al. Independent prognostic value of cardiac troponin $T$ in patients with confirmed pulmonary embolism. Circulation. 2000;102(2):211-217.

18. Jardin F, Dubourg O, Bourdarias J-P. Echocardiographic pattern of acute cor pulmonale. Chest. 1997;111(1):209-217.

19. Zakynthinos E, Zakynthinos S. Contemporary diagnosis and therapy of pulmonary hypertension. Hellenic J Cardiol. 1991;32:111-123.

20. Eid-Lidt G, Gaspar J, Sandoval J, et al. Persistent pulmonary hypertension and right ventricular function after percutaneous mechanical thrombectomy in severe acute pulmonary embolism. Eur Respir J 2017; 49: 1600910

21. Jennifer Cowger Matthews, Vallerie McLaughlin. Acute Right Ventricular Failure in the Setting of Acute Pulmonary Embolism or Chronic Pulmonary Hypertension: A Detailed Review of the Pathophysiology, Diagnosis, and Management. Curr Cardiol Rev. 2008 Feb; 4(1): 49-59.

22. Darze ES, Casqueiro JB, Ciuffo LA, Santos JM, Magalhães IR, Latado AL. Pulmonary embolism mortality in Brazil from 1989 to 2010: Gender and regional disparities. Arquivos Brasileiros de Cardiologia. 2016;106(1):4-12.

23. Khemasuwan D, Yingchoncharoen $T$, Tunsupon $P$, et al. Right ventricular echocardiographic parameters are associated with mortality after acute pulmonary embolism. J Am Soc Echocardiogr. Mar 2015;28(3):355-62. doi:10.1016/j.echo.2014.11.012

24. Kurnicka K, Lichodziejewska B, Goliszek S, et al. Echocardiographic Pattern of Acute Pulmonary Embolism: Analysis of 511 Consecutive Patients. J Am Soc Echocardiogr. Sep 2016;29(9):907-13. doi:10.1016/j.echo.2016.05.016

25. Bajaj N, Bozarth AL, Guillot J, et al. Clinical features in patients with pulmonary embolism at a community hospital: analysis of 4 years of data. Journal of thrombosis and thrombolysis. 2014;37(3):287-292.

26. Grifoni S, Olivotto I, Cecchini P, et al. Short-term clinical outcome of patients with acute pulmonary embolism, normal blood pressure, and echocardiographic right ventricular dysfunction. Circulation. Jun 20 2000;101(24):2817-22. doi:10.1161/01.cir.101.24.2817

27. Matthews JC, McLaughlin V. Acute right ventricular failure in the setting of acute pulmonary embolism or chronic pulmonary hypertension: a detailed review of the pathophysiology, diagnosis, and management. Curr Cardiol Rev. Feb 2008;4(1):49-59. doi:10.2174/157340308783565384 
28. Wood KE. Major pulmonary embolism: review of a pathophysiologic approach to the golden hour of hemodynamically significant pulmonary embolism. Chest. Mar 2002;121(3):877-905. doi:10.1378/chest.121.3.877

29. Ende-Verhaar YM, Cannegieter SC, Vonk Noordegraaf A, et al. Incidence of chronic thromboembolic pulmonary hypertension after acute pulmonary embolism: a contemporary view of the published literature. Eur Respir J. Feb 2017;49(2)doi:10.1183/13993003.01792-2016

\section{Tables}

Table 1

Patients' characteristics and clinical features

\begin{tabular}{|lll|}
\hline & $\begin{array}{l}\text { Number } \\
\text { (Total = 183) }\end{array}$ & percent \\
\hline Sex male & 83 & 45.4 \\
female & 100 & 54.6 \\
\hline Age $<40$ y & 29 & 15.8 \\
40-60 y & 48 & 26.2 \\
60-70 y & 37 & 20.2 \\
$>70$ y & 69 & 37.7 \\
\hline Dyspnea & 161 & 88 \\
\hline Chest pain & 90 & 49.2 \\
\hline Shock state & 68 & 37.2 \\
\hline Hemoptysis & 24 & 13.1 \\
\hline Syncope & 13 & 7.1 \\
\hline Tachycardia & 99 & 54.1 \\
\hline Tachypnea & 99 & 54.1 \\
\hline
\end{tabular}


Table 2

Echocardiographic findings of the patients and its relationship with in hospital outcomes

\begin{tabular}{|lllll|}
\hline & number & percent & death & P value \\
\hline TR severity normal & 23 & 12.6 & 2 & 0.007 \\
mild & 65 & 35.5 & 5 & \\
moderate & 52 & 28.4 & 13 & \\
severe & 43 & 23.5 & 17 & \\
RV dysfunction present & 124 & 67.8 & 30 & 0.03 \\
absent & 59 & 32.2 & 7 & \\
sPAP normal & 45 & 24.6 & 3 & 0.002 \\
30-40 & 19 & 10.4 & 0 & \\
$40-60^{\text {mmh }}$ & 42 & 23 & 11 & \\
$>60^{\text {mmhg }}$ & 77 & 42.1 & 23 & \\
\hline
\end{tabular}

Table 3

Relationship between pulmonary artery pressure severity and shock state

\begin{tabular}{|c|c|c|}
\hline & $\begin{array}{l}\text { Shock state } \\
(\text { Total }=68)\end{array}$ & $P$ value \\
\hline sPAP normal & 10(14.7\%) & 0.006 \\
\hline $30-40^{\mathrm{mmhg}}$ & $3(4.4 \%)$ & \\
\hline $40-60^{\mathrm{mmhg}}$ & $18(26.5 \%)$ & \\
\hline$>60^{\mathrm{mmhg}}$ & $37(54.4)$ & \\
\hline
\end{tabular}

